\title{
Global Assimilation of Ionospheric Measurements (GAIM)
}

Robert W. Schunk, ${ }^{1}$ Ludger Scherliess, ${ }^{1}$ Jan J. Sojka, ${ }^{1}$ Donald C. Thompson, ${ }^{1}$ David N. Anderson, ${ }^{2}$ Mihail Codrescu, ${ }^{2}$ Cliff Minter, ${ }^{2}$ Timothy J. Fuller-Rowell, ${ }^{2}$ Roderick A. Heelis, ${ }^{3}$ Marc Hairston, ${ }^{3}$ and Bruce M. Howe ${ }^{4}$

Received 9 October 2002; revised 26 March 2003; accepted 1 May 2003; published 31 January 2004.

[1] The ionosphere is a highly dynamic medium that exhibits weather disturbances at all latitudes, longitudes, and altitudes, and these disturbances can have detrimental effects on both military and civilian systems. In an effort to mitigate the adverse effects, we are developing a physics-based data assimilation model of the ionosphere and neutral atmosphere called the Global Assimilation of Ionospheric Measurements (GAIM). GAIM will use a physics-based ionosphere-plasmasphere model and a Kalman filter as a basis for assimilating a diverse set of real-time (or near real-time) measurements. Some of the data to be assimilated include in situ density measurements from satellites, ionosonde electron density profiles, occultation data, ground-based GPS total electron contents (TECs), two-dimensional ionospheric density distributions from tomography chains, and line-of-sight UV emissions from selected satellites. When completed, GAIM will provide specifications and forecasts on a spatial grid that can be global, regional, or local. The primary output of GAIM will be a continuous reconstruction of the three-dimensional electron density distribution from $90 \mathrm{~km}$ to geosynchronous altitude $(35,000 \mathrm{~km})$. GAIM also outputs auxiliary parameters, including $N_{m} F_{2}, h_{m} F_{2}, \mathrm{~N}_{\mathrm{m}} \mathrm{E}, \mathrm{h}_{\mathrm{m}} \mathrm{E}$, and slant and vertical TEC. Furthermore, GAIM provides global distributions for the ionospheric drivers (neutral winds and densities, magnetospheric and equatorial electric fields, and electron precipitation patterns). In its specification mode, GAIM yields quantitative estimates for the accuracy of the reconstructed ionospheric densities. INDEX TERMS: 2447 Ionosphere: Modeling and forecasting; 2467 Ionosphere: Plasma temperature and density; 2415 Ionosphere: Equatorial ionosphere; 2443 Ionosphere: Midlatitude ionosphere; 2475 Ionosphere: Polar cap ionosphere; KEYWORDS: ionosphere, assimilation, Kalman filter, plasmasphere

Citation: Schunk, R. W., et al. (2004), Global Assimilation of Ionospheric Measurements (GAIM), Radio Sci., 39, RS1S02, doi:10.1029/2002RS002794.

\section{Introduction}

[2] The ionosphere has been studied extensively for more than 50 years, and it is now well known that the ionosphere exhibits a significant variation with altitude, latitude, longitude, universal time, solar cycle, season, and geomagnetic activity. This variation results from the

\footnotetext{
${ }^{1}$ Center for Atmospheric and Space Sciences, Utah State University, Logan, Utah, USA.

${ }^{2} \mathrm{NOAA} /$ Space Environment Center, Department of Commerce, Boulder, Colorado, USA.

${ }^{3}$ W. B. Hanson Center for Space Sciences, University of Texas at Dallas, Richardson, Texas, USA.

${ }^{4}$ Applied Physics Laboratory, University of Washington, Seattle, Washington, USA.
}

Copyright 2004 by the American Geophysical Union.

0048-6604/04/2002RS002794\$11.00 various chemical, transport, and radiative processes that operate in the ionosphere-thermosphere system as well as from the effects of solar, interplanetary, magnetospheric, and mesospheric processes. The various processes act to create both a background ionospheric state (climatology) and a disturbed state (weather). The ionospheric features that are associated with the background state include a tongue of ionization in the polar cap, a polar hole in winter, enhanced densities in the auroral oval, elevated dayside densities, a midlatitude trough at night, and the equatorial ionization anomaly. However, superimposed on these background ionospheric features is a wide range of weather disturbances, and as a consequence, the ionosphere can display significant hourly and daily variations.

[3] These weather disturbances can have detrimental effects on numerous human activities and systems. They can adversely affect survey and navigation systems that use Global Positioning System (GPS) satellites, over-the- 


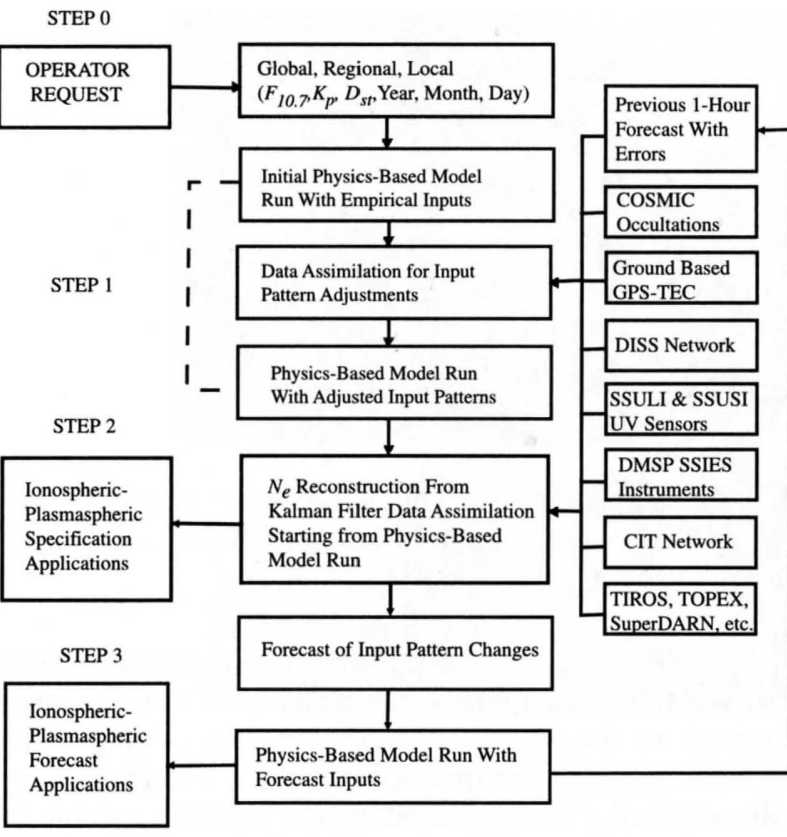

Figure 1. Block diagram of the GAIM specification and forecast approach. See color version of this figure at back of this issue.

horizon (OTH) radars, HF communications, surveillance, satellite tracking and lifetimes, power grids, pipelines, and the Federal Aviation Administration's Wide-Area Augmentation System (WAAS). In an effort to mitigate the adverse effects of the ionosphere on military and civilian systems, specification and forecast models are being used both to correct for ionospheric effects and to predict weather disturbances. Currently, numerous modeling approaches are being used in various space weather applications, including empirical models, analytical and parameterized models, global numerical models that couple different spatial domains (magnetosphere, plasmasphere, ionosphere, thermosphere) and data assimilation models. Also, hybrid models are being used whereby different model types are combined for practical purposes.

[4] The most promising ionospheric weather models are the physics-based data-driven models that use Kalman filter data assimilation techniques. Although such techniques have been successfully used by the meteorologists and oceanographers for several decades, the space physics community has been slow in implementing data assimilation techniques, primarily because of the lack of a sufficient number of measurements. However, this situation is changing rapidly for the ionosphere. Within 10 years, it is anticipated that there will be several million ionospheric measurements per day from a variety of sources, and these data will be available for assimilation into specification and forecast models. Because of this possibility, we have begun the development of a physics-based, Kalman filter, data assimilation model of the ionosphere. This paper describes an overview of our approach and the current status of the model.

\section{Global Assimilation of Ionospheric Measurements (GAIM)}

[5] GAIM uses a time-dependent physics-based model of the global ionosphere-plasmasphere and a Kalman filter as a basis for assimilating a diverse set of real-time (or near real-time) measurements. When completed, GAIM will provide both specifications and forecasts on a spatial grid that can be global, regional, or local. The primary output of GAIM will be a continuous reconstruction of the three-dimensional $\mathrm{N}_{\mathrm{e}}$ distribution from $90 \mathrm{~km}$ to $35,000 \mathrm{~km}$. However, GAIM will also provide a range of auxiliary parameters as well as the main ionospheric drivers. In its specification mode, GAIM will give quantitative estimates for the accuracy of the reconstructed ionospheric densities. Also, GAIM will have a modular construction, so that when new models, observing stations, and data types become available, they can be easily incorporated into the data assimilation scheme. The overall program involves model construction, data quality assessment, data assimilation, the construction of an executive system to automatically run GAIM in real time, and validation. In the subsections that follow, we first describe the GAIM system and then we describe the various elements of GAIM in more detail.

\subsection{GAIM System}

[6] Figure 1 shows a block diagram of the GAIM approach to ionospheric specifications and forecasts. GAIM is a four-level system that provides time-dependent electron density distributions regardless of the amount of data available for assimilation.

[7] Step 0 corresponds to time-dependent climatology and it provides the ionospheric specification if no, or too little, data are available. The specification is obtained from the physics-based Ionospheric Forecast Model (IFM), which is currently operational at the Air Force Weather Agency (AFWA) in Omaha, Nebraska. The ionospheric drivers (neutral densities and winds, magnetospheric and equatorial electric fields, auroral precipitation) needed by the IFM are self-contained in the model and are given by well-known empirical models. The IFM is run by specifying a few simple geophysical parameters (year, day, start time, duration of run, $\mathrm{F}_{10.7}, K_{p}$ ). The IFM's output is global $N_{e}$ distributions from 90 to $1600 \mathrm{~km}$ as a function of time.

[8] In step 1, selected data from the observational database are used to adjust the empirical drivers so that the drivers are consistent with the measurements, and 


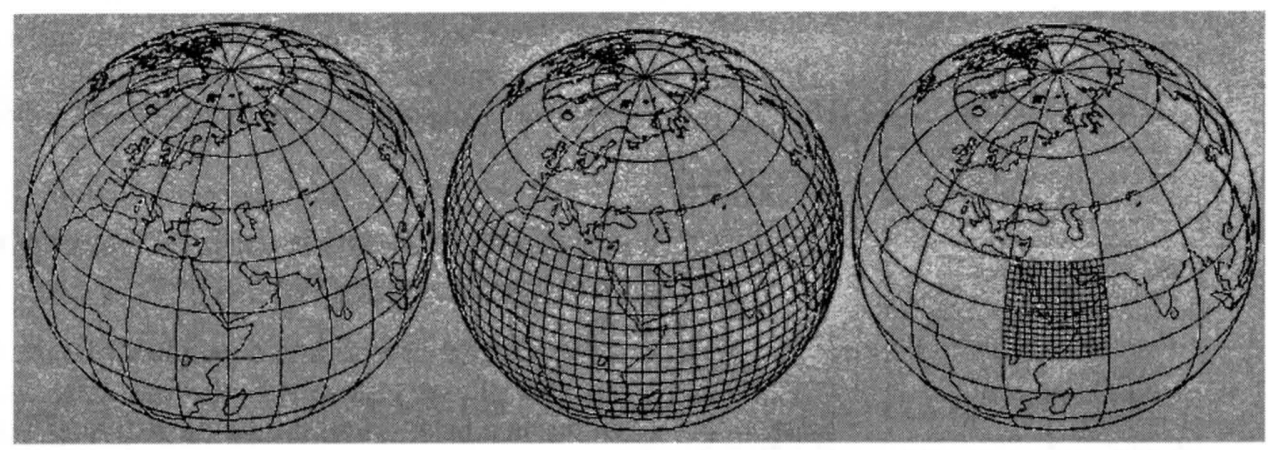

Figure 2. The default spatial grids used in GAIM. There is a global grid (left), a higher-resolution regional grid (middle), and a fine-resolution local grid. See color version of this figure at back of this issue.

then a physics-based Ionosphere-Plasmasphere Model (IPM) is run using the adjusted drivers. Because measured drivers are used, the global $N_{e}$ distribution obtained at this step should be an improvement over that obtained at step 0 . Further details concerning the adjustment of the ionospheric drivers are given in section 2.6.

[9] For step 2, the $N_{e}$ distribution obtained from the step 1 ionosphere-plasmasphere simulation is used as a starting point (first guess) for a true electron density reconstruction using all of the different data types that pertain to $N_{e}$. An approximate Kalman filter is used to accomplish the data assimilation. After the assimilation process is started, the Kalman filter combines the simulation results from the physics-based ionosphere-plasmasphere model with the available real-time data to produce a continuous reconstruction of the global $N_{e}$ distribution from $90-35,000 \mathrm{~km}$.

[10] The motivation for starting from the step $1 N_{e}$ distribution is that it should be close to the real $N_{e}$ distribution, because it is based on measured inputs (drivers). If the first guess is close to the real $N_{e}$ distribution (e.g., reasonably correct density gradients and features), the Kalman filter reconstruction is more likely to converge to the correct $N_{e}$ distribution. Note that although step 1 is only needed to start the Kalman filter $N_{e}$ reconstruction, both steps 0 and 1 are also run continuously, because a comparison of the three $N_{e}$ distributions at each time step provides information about the errors associated with the $N_{e}$ reconstruction. Therefore our assimilation procedure provides quantitative error estimates for each reconstruction, does not introduce artificial density gradients, and provides a means of ionospheric forecasting.

[11] Finally, it should be noted that in an operational setting the three GAIM levels (steps 0,1 , and 2) will be transparent to the user. Only the Kalman filter $N_{e}$ reconstruction (step 2) will be available for applications/products. Step 2 will correspond to the best specification of the ionosphere-plasmasphere system.
[12] Step 3 corresponds to the GAIM forecast mode. At a given time, the reconstructed $N_{e}$ distribution corresponds to the specification of the ionosphere-plasmasphere system at that time. Using this $N_{e}$ distribution, and the adjusted global input patterns, it is possible to run the physics-based model and produce forecasts for the ionosphere-plasmasphere system. Persistence forecasts are obtained by keeping the global inputs fixed and then running the physics-based model forward in time. However, it is possible to do much better than this, because it is possible to forecast the global inputs. Most of them are linked in some way to magnetic activity changes (e.g., $K_{p}$ ), and we have already developed a forecast algorithm for $K_{p}$ that is much better than persistence. This $K_{p}$ forecast algorithm can be used to obtain forecasts for the convection electric field, particle precipitation, and neutral wind patterns. With these forecasted inputs, it should be possible to provide reliable near-term ionospheric forecasts, although this work has just begun.

\subsection{Output Grid}

[13] The output grid in GAIM is designed to be flexible (Figure 2). Depending on the application, the grid can be global, regional, or local $(25 \mathrm{~km} \times 25 \mathrm{~km})$. Typically, smaller output grids yield a better spatial resolution, but in reality, the actual resolution is determined by the available data. GAIM also has the ability to assimilate data from a large number of newly created data sources. For example, if GPS receivers are placed in or around a specific country or area, the receiver locations can be entered into GAIM and the program will automatically assimilate the slant path TECs obtained from the new receivers. An application of this nature should yield a high-resolution ionospheric reconstruction over the selected country or area.

\subsection{Data Sources}

[14] GAIM has the ability to assimilate a wide range of data types from numerous ground-based locations and 


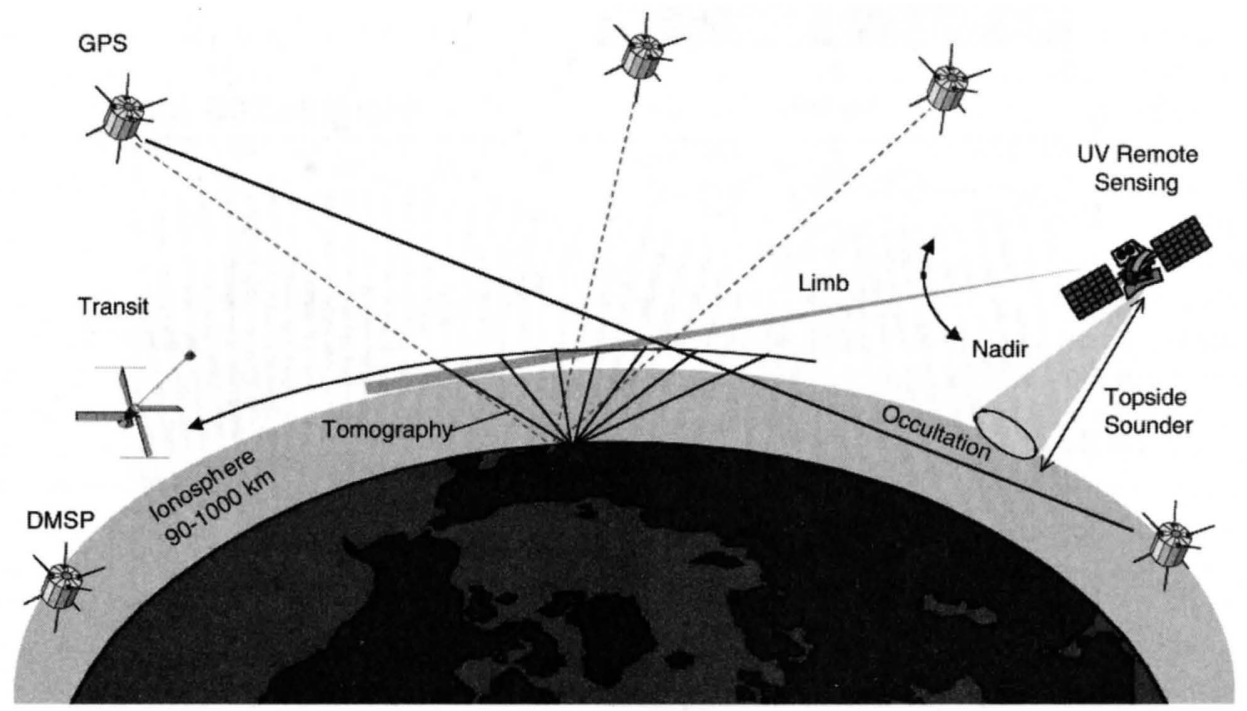

Figure 3. Schematic diagram showing some of the data sources that are available for assimilation into GAIM. Adapted from McCoy [2001]. See color version of this figure at back of this issue.

space-based platforms. Figure 3 is a schematic of some of the data sources that are, or could be, available for assimilation in GAIM during the next decade. The data sources include in situ electron densities from NOAA and DoD operational satellites, bottomside electron density profiles from a network of 100 Digisondes, line-ofsight total electron content (TEC) measurements between as many as several thousand ground stations and the GPS satellites, TECs between low-altitude satellites with radio beacons and several ground-based tomography chains, TECs via occultations between various low-altitude satellites and between low- and high-altitude satellites, line-of-sight UV emission data, and magnetometer data from a network of 100 ground stations.

[15] To be useful for a model that will provide continuous ionospheric specifications, the data must be in real time or in near real time (within $90 \mathrm{~min}$ of the specification). Also, the uncertainty in the data must be known, because this is an input to the Kalman filter analysis. Furthermore, since the data assimilation will be in real time, software is required to detect and eliminate bad data, to fill data gaps, and to account for data outages. In addition, procedures must be established to independently analyze data streams for long-term problems, such as changes in biases and instrument degradation.

\subsection{Physics-Based Models}

[16] The Ionosphere Forecast Model (IFM) is a physics-based numerical model of the global ionosphere [Schunk et al., 1997]. The model calculates three-dimensional, time-dependent density distributions for four major ions $\left(\mathrm{NO}^{+}, \mathrm{O}_{2}^{+}, \mathrm{N}_{2}^{+}, \mathrm{O}^{+}\right)$at $E$ region altitudes, two major $\left(\mathrm{O}^{+}, \mathrm{NO}^{+}\right)$and two minor $\left(\mathrm{N}_{2}^{+}, \mathrm{O}_{2}^{+}\right)$ions at $F$ region altitudes, and the ion and electron temperatures at both $E$ and $F$ region altitudes. The IFM also contains a simple prescription for calculating $\mathrm{H}^{+}$densities in the $F$ region and topside ionosphere. The model covers the altitude range from 90 to $1600 \mathrm{~km}$, and outputs density values at a spatial resolution of $4 \mathrm{~km}$ in the $E$ region and $20 \mathrm{~km}$ in the $F$ region. The model outputs the density and temperature distributions in either a geographic or geomagnetic coordinate system with a $3^{\circ}$ latitude resolution and a $7.5^{\circ}$ longitude resolution. The IFM is selfcontained and easy to use, being driven by a few simple geophysical indices. The model drivers include $F_{10.7}$, year, day, start time, duration of the model run, and the temporal variation of $K_{p}$ from 3 hours prior to the start time to the end of the simulation.

[17] The IFM is based on a numerical solution of the ion and electron continuity, momentum, and energy equations. The model takes account of the following physical processes: (1) Field-aligned diffusion due to density and temperature gradients, gravity, and the ambipolar electric field; (2) cross-field electrodynamic drifts due to both magnetospheric and dynamo electric fields; (3) ion production due to UV and EUV solar radiation, resonantly scattered solar radiation, starlight, and auroral electron precipitation; (4) numerous energydependent chemical reactions; (5) thermospheric winds; (6) neutral composition changes; (7) thermal conduction; and (8) a host of elastic and inelastic heating and cooling processes. The IFM also takes account of the offset between the geomagnetic and geographic poles. 

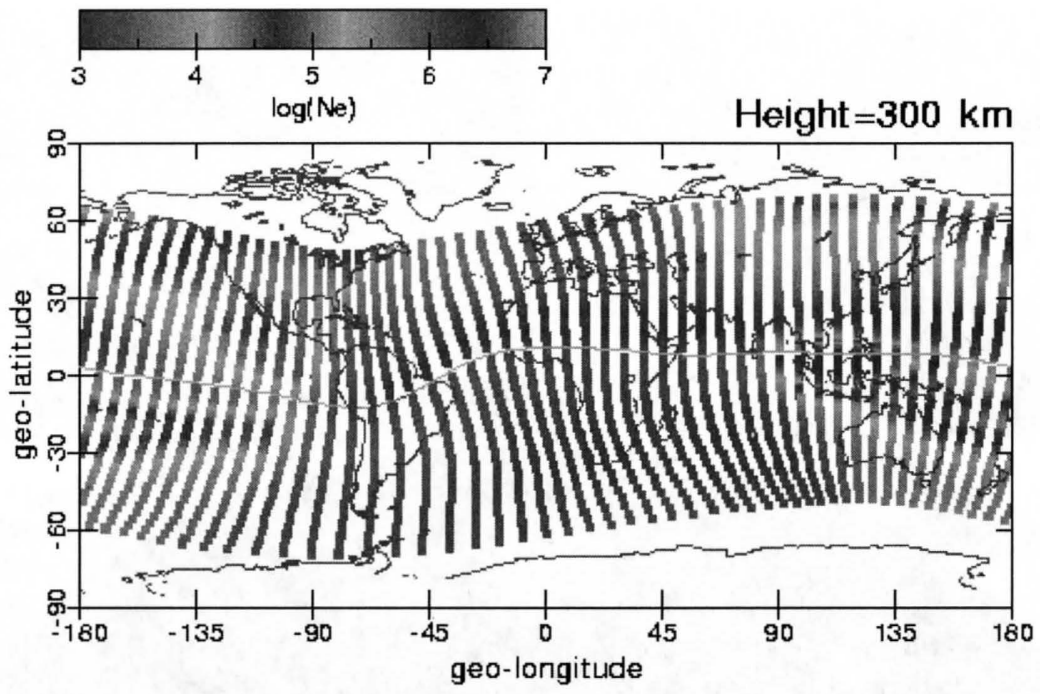

Figure 4. Snapshot of modeled electron densities at $300 \mathrm{~km}$ and 12 UT displayed in a geographic coordinate system. The electron densities were calculated from the IPM and are shown along geomagnetic field lines. See color version of this figure at back of this issue.

[18] The Ionosphere-Plasmasphere Model (IPM) was developed specifically for data assimilation purposes, where access to individual plasma flux tubes is useful [Schunk, 2002]. The IPM covers geomagnetic latitudes from about $60^{\circ} \mathrm{N}$ to $60^{\circ} \mathrm{S}$ and equatorial crossing altitudes from 90 to $35,000 \mathrm{~km}$. The IPM includes chemical, radiative, and transport processes that are similar to those in the IFM, but the IPM also self-consistently includes $\mathrm{H}^{+}$. At $E$ region altitudes, chemical equilibrium is assumed and the continuity equations for $\mathrm{NO}^{+}, \mathrm{O}_{2}^{+}$, $\mathrm{N}_{2}^{+}$, and $\mathrm{O}^{+}$are solved simultaneously at each grid point for the ion densities. At $F$ region altitudes and above, an Euler-Lagrange hybrid numerical scheme is used. The continuity and momentum equations for $\mathrm{H}^{+}$and $\mathrm{O}^{+}$are solved along dipolar magnetic field lines for individual plasma flux tubes taking into account equatorial electric fields and interactions with the neutral atmosphere. The field-aligned transport equations are first transformed to spherical coordinates, then dipolar coordinates, and finally to a "sinh" variable [Schunk and Nagy, 2000, chap. 11]. These transformations yield an unequal grid spacing along $\mathbf{B}$ that is different for each field line, but these transformations provide for an efficient numerical solution. Next, the dipolar field lines are adjusted to agree with the International Geomagnetic Reference Field (IGRF), so that they have the same apex altitudes and longitudinal variations. Finally, the plasma flux tubes are followed as they convect through a moving neutral atmosphere perpendicular to $\mathbf{B}$ due to corotational and dynamo electric fields. The three-dimensional nature of the model is obtained by following many plasma flux tubes while keeping track of their positions at all times.

[19] Both the IFM and IPM models require certain inputs, including the atmospheric densities and winds, the magnetospheric and dynamo electric fields, and the auroral electron precipitation. In general, these inputs need to be global and time-dependent. For the IFM, the required inputs are included as an integral part of the model via empirical models. The adopted empirical models are: the MSIS-90 model for the atmospheric densities [Hedin, 1991]; the HWM for the neutral winds [Hedin et al., 1991]; the Weimer [1995] model for the magnetospheric electric fields; the Hardy et al. [1985] model for the electron precipitation; and the Scherliess and Fejer [1999] model for the equatorial electric fields.

[20] The IPM can also be driven by empirical drivers, and in this case an empirical model for $T_{e}$ and $T_{i}$ is needed, because the energy equations are not solved. The Titheridge [1998] model is adopted for $T_{e}$ and $T_{i}$ in the ionosphere and plasmasphere. However, the real advantages of the IPM are that it can be used to deduce the ionospheric drivers and that it can be used efficiently in the Kalman filter data assimilation scheme.

[21] Figure 4 shows IPM results for the case when empirical ionospheric drivers are used. The figure shows a snapshot of the global electron density at $300 \mathrm{~km}$ and 1200 UT for day 336 in 1998 . The conditions correspond to relatively quiet magnetic activity $\left(K_{p}=2\right)$ and moderate solar activity $\left(F_{10.7}=158\right)$. The electron densities are shown along geomagnetic field lines from $-60^{\circ}$ to 
$+60^{\circ}$ magnetic; but they are displayed in a geographic coordinate system. Clearly evident is the strong influence of the geomagnetic field on $N_{e}$ at low latitudes. This feature must be properly accounted for in any data assimilation model.

\subsection{Kalman Filter}

[22] GAIM uses a Kalman filter technique for the main data assimilation algorithm, which continuously reconstructs the global electron density. This filter provides an efficient means for assimilating different data types into a time-dependent, physics-based, numerical model, taking into account the uncertainties in both the model and data [Daley, 1991]. The Kalman filter is a sequential least squares procedure that finds the best estimate of the state (ionosphere-plasmasphere system) at time t based on all the information that is available prior to this time. The basic principle is to combine measurements from an observational network with the information obtained from a physics-based ionospheric-plasmasphere model, taking into account the corresponding statistical description of uncertainties. Formally, the Kalman filter performs a recursive least squares inversion of all of the measurements (slant TEC, in situ satellite, Digisonde, etc.) for the model variable (electron density) using the physics-based model as a constraint. In practice, a weighted average of the measurements and the model result is taken, using the relative accuracy of the two as the weights. The net result is an improved estimate of the model variable (electron density), where the improvement is in a statistical sense. The improved estimate for $N_{e}$ has the least expected error given the measurements, model, and error statistics. With this approach, the specification of the error covariances for both the measurements and model is critical. This issue and the mathematical details associated with the Kalman filter are given in a companion paper by Scherliess et al. [2004].

\subsection{Driver Determination}

[23] In step 1, selected data from the observational database are used to adjust the empirical models for the ionospheric drivers so that they agree with the measurements. The philosophy here is that certain data sources are more reliable than others when one wants to deduce the ionospheric drivers. For example, at midlatitudes the meridional neutral wind is the main input needed by the physics-based ionospheric-plasmasphere model, and since $h_{m} F_{2}$ is sensitive to the meridional wind, Digisonde data are more useful than, for example, slant TEC for deducing the wind.

[24] As noted above, the main ionospheric drivers are the neutral densities and winds, the magnetospheric and equatorial electric fields, and auroral precipitation. When measurements are available that relate to these drivers, empirical models are adjusted to bring agreement between models and measurements. Briefly, this is accomplished as follows: (1) DMSP satellite measurements of electron precipitation and plasma drifts are used to adjust the empirical models of Hardy et al. [1985] and Weimer [1995], respectively [Bekerat et al., 2001]. This procedure yields time-dependent precipitation and convection patterns; (2) DIGISONDE and other data that relate to the neutral wind provide information at specific locations and these individual measurements are used in conjunction with a vector spherical harmonic expansion to obtain an adjusted global wind pattern [Jee et al., 2001]; (3) line-of-sight UV data are assimilated into a model of the neutral atmosphere via a Kalman filter to obtain a global map of the neutral composition $\left(\mathrm{O} / \mathrm{N}_{2}\right.$ ratio) as a function of time [Fuller-Rowell et al., 2002]; and (4) magnetometer measurements during the daytime and Digisonde data at night are used to determine the equatorial electric field at specific locations as a function of time [Anderson et al., 2002], and these data are used to adjust the Scherliess and Fejer [1999] empirical model of equatorial electric fields. Note that preliminary algorithms are already available to accomplish the above. However, GAIM is modular and as the various algorithms are updated with additional data sources they can be easily inserted into GAIM without affecting the rest of the system.

\section{GAIM Simulation}

[25] GAIM is an extensive system that is still under development. When completed, GAIM will contain many different codes and algorithms, and they all must be validated and tested for robustness. Consequently, in the initial development phase, the physics-based ionosphere-plasmasphere model was not used in the Kalman filter data assimilation scheme. Instead, a simple GaussMarkov model was adopted. Also, in this initial work, synthetic data (with noise) were used so we could verify that the relevant algorithms were working correctly. This initial work is described by Schunk et al. [2003]. Next, the synthetic data were replaced with real data, but the simple Gauss-Markov model was still used. This latter effort is described in this section. The third phase of our work involved the use of the physics-based ionosphereplasmasphere model in the Kalman filter, but synthetic data were used. This latter work is briefly mentioned in section 4 of this paper and is described in more detail in a companion paper by Scherliess et al. [2004]. At the present time, we are in the process of using real data with the physics-based Kalman filter model.

[26] For the GAIM simulation presented here, a continuous 3-day stream of data from several sources was used to test the initial GAIM system. The data were for the period 2-5 December 1998 (days 336-338) and 
IRI $199833600: 00$
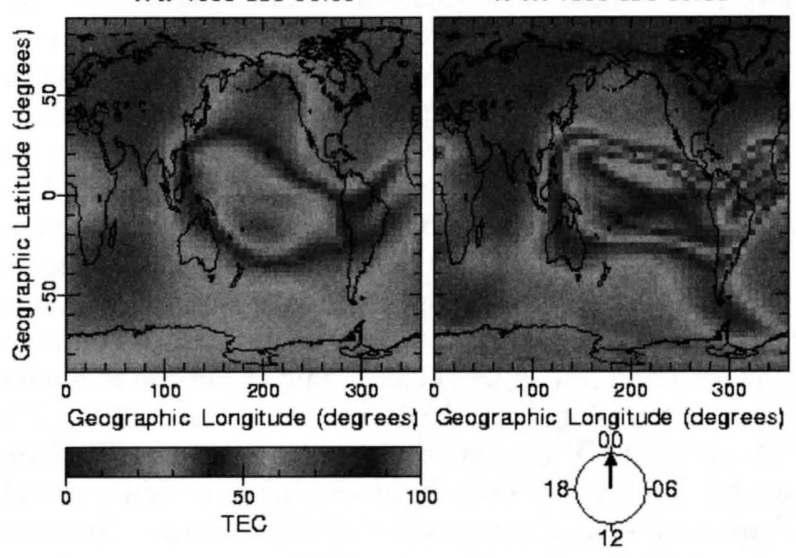

Figure 5. Snapshots of the vertical TEC distributions obtained from the (left) IRI and the (right) IFM at 0:00 UT shown in a geographic coordinate system. The conditions are for day 336 in 1998. Note that the IFM corresponds to GAIM-step 0. See color version of this figure at back of this issue.

included measurements from 2 DMSP satellites, 42 ground-based GPS receivers, and 16 Digisondes. The database also included the temporal variation of $\mathrm{F}_{10.7}$ and $K_{p}$, and magnetometer data from two equatorial stations. In general, $F_{10.7} \sim 150$ and $K_{p} \sim 1-3$ during this 3-day period. Note that the simulation was restricted to only these data sources because they are the main data types currently available to the Air Force Weather Agency in real time, and hence, they would be available for a data assimilation model like GAIM. Also, for this simulation, all three levels of GAIM (steps 0-2) were run simultaneously. The complete results of this GAIM simulation are given in a $C D$ that can be obtained from the first author of this paper. The CD contains several movies of the 3-day simulation, including movies of the data-driven modified drivers, the adaptive coordinate system, and the results from steps 0,1 , and 2 of GAIM. In what follows, only a brief synopsis of the 3-day simulation is given.

[27] Step 0 of GAIM corresponds to time-dependent climatology. At this step, the geophysical indices for the 3-day period are used to drive the Ionospheric Forecast Model (IFM). Figure 5 shows a snapshot of the global distribution of vertical TEC obtained from the IFM by integrating $\mathrm{N}_{\mathrm{e}}$ from 90 to $1600 \mathrm{~km}$. The snapshot is for day 336 at 0000 UT. Figure 5 also shows the corresponding result from the International Reference Ionosphere (IRI) for comparison. Note that at step 0, GAIM is already superior to the IRI at high and low latitudes, as shown by previous validations of the IFM.

[28] At step 1, selected data are used to obtain modified ionospheric drivers and then these drivers are used to rerun the IFM, which yields an improved ionospheric specification. In GAIM, the run is automatic and continuous; the data are acquired, the modified drivers are determined, and the IFM is run. For this simulation, the DMSP particle precipitation and plasma drift measurements were used to obtain modified auroral oval and plasma convection patterns for both the Northern and Southern Hemispheres for the 3-day period. Also, the magnetometer data were used to obtain a modified longitudinal distribution of the equatorial electric field. These time-dependent ionospheric drivers were then automatically used to run the IFM, and a snapshot of the results is shown in Figure 6. The figure shows the global distribution of vertical TEC at 20:00 UT (GAIMstep1-modified drivers). Also shown is the corresponding result from GAIM 0 (empirical drivers). There were significant differences between the step 0 and 1 results during the 3-day period, as expected, and the step 1 results are an improvement over the step 0 results, based on previous validations. The validation of this specific simulation is currently in progress.

[29] At step 2, all of the measurements in the database that pertain to $\mathrm{N}_{\mathrm{e}}$ are used in the Kalman filter assimilation scheme starting from the $\mathrm{N}_{\mathrm{e}}$ distribution obtained in step 1. However, after this data assimilation scheme is started (at a specified time), the Kalman filter combines the simulation results from an ionospheric model with the real-time measurements to produce a continuous reconstruction of the global $\mathrm{N}_{\mathrm{e}}$ distribution as a function of time. The data sources that are typically included in our Kalman filter reconstructions are shown in Figure 7. There are 16 Digisondes, 42 ground-based GPS receivers, and 2 DMSP satellites, although in the results

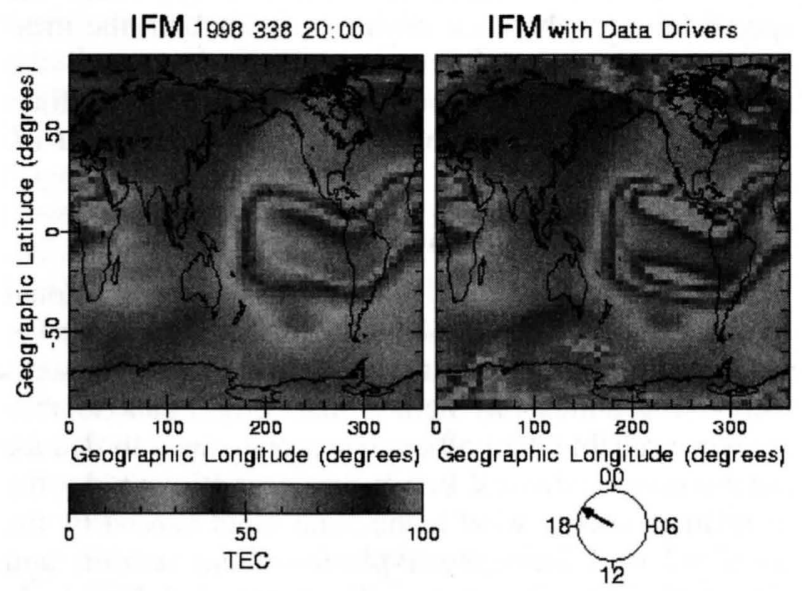

Figure 6. Snapshots of the vertical TEC distributions obtained from the IFM (GAIM-step 0) and the IFM with data-driven ionospheric drivers (GAIM-step 1) at 20 UT shown in a geographic coordinate system. The conditions are for day 338 in 1998. See color version of this figure at back of this issue. 


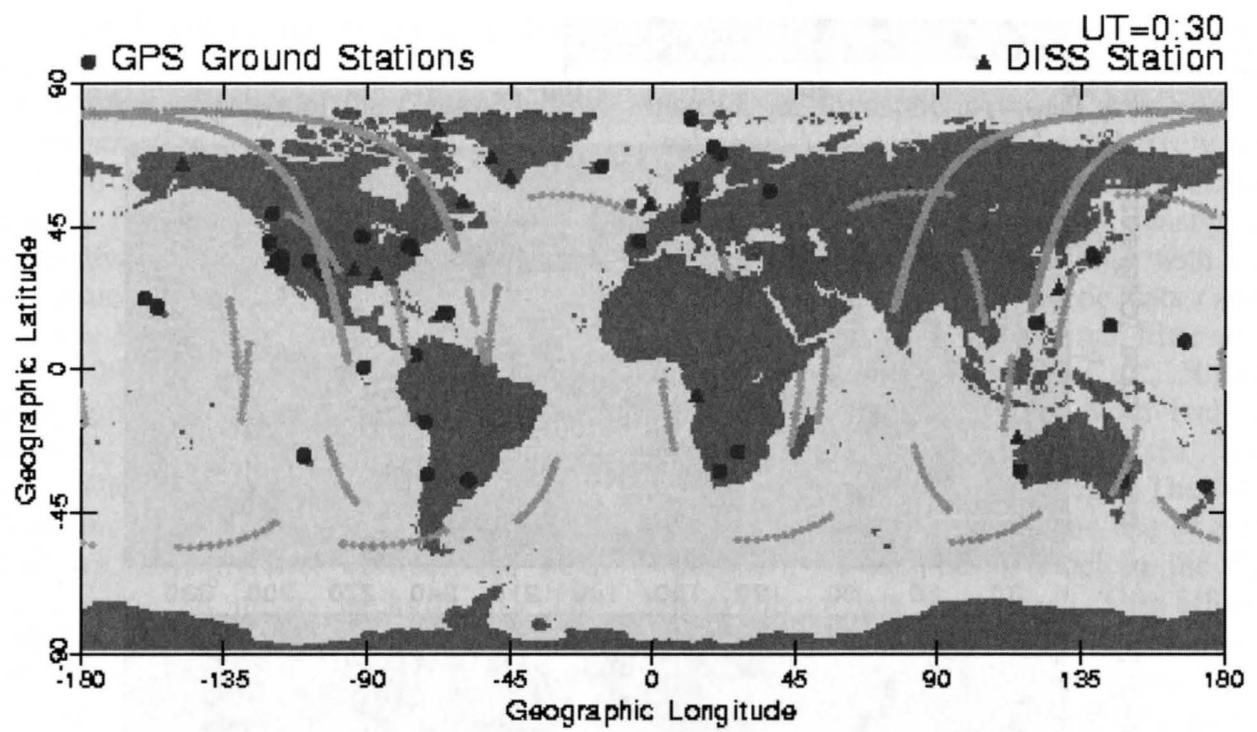

Figure 7. Data sources used in the Gauss-Markov Kalman filter electron density reconstruction. There are 16 Digisondes (triangles), 42 ground-based GPS receivers (circles) and 2 DMSP satellites. The short curved lines show the GPS receiver/satellite field-of-views for a 1-hour period. The long curved lines show the DMSP satellite tracks for a 1-hour period. The configuration is for 0:30 UT. See color version of this figure at back of this issue.

to be shown later only one of the 16 Digisondes was used. The data are assimilated exactly as they are measured, i.e., bottomside $\mathrm{N}_{\mathrm{e}}$ profiles from the Digisondes, in situ $\mathrm{N}_{\mathrm{e}}$ along the DMSP satellite tracks, and slant TEC from GPS ground-based receivers. Note that the instrument configuration shown in Figure 7 corresponds to a snapshot at 0030 UT on day 336. As the Earth rotates, the satellite tracks and TEC fields of view move around, but this is automatically taken into account in the Kalman filter analysis.
[30] As noted above, the physics-based ionospheric model was not used in the Kalman filter. Instead, a first-order Gauss-Maukov process was used to describe the change in electron density, $\Delta \mathrm{N}_{\mathrm{e}}$, due to the measurements. The change $\Delta \mathrm{N}_{\mathrm{e}}$ was implemented as $\exp (-t / \tau)$, where $t$ is the assimilation time step and $\tau$ is a time constant. Specifically, the transition matrix was taken to be diagonal with all the terms equal to $\exp (-t / \tau)$. In the simulation, the IFM was used to describe the background ionosphere and the Kalman
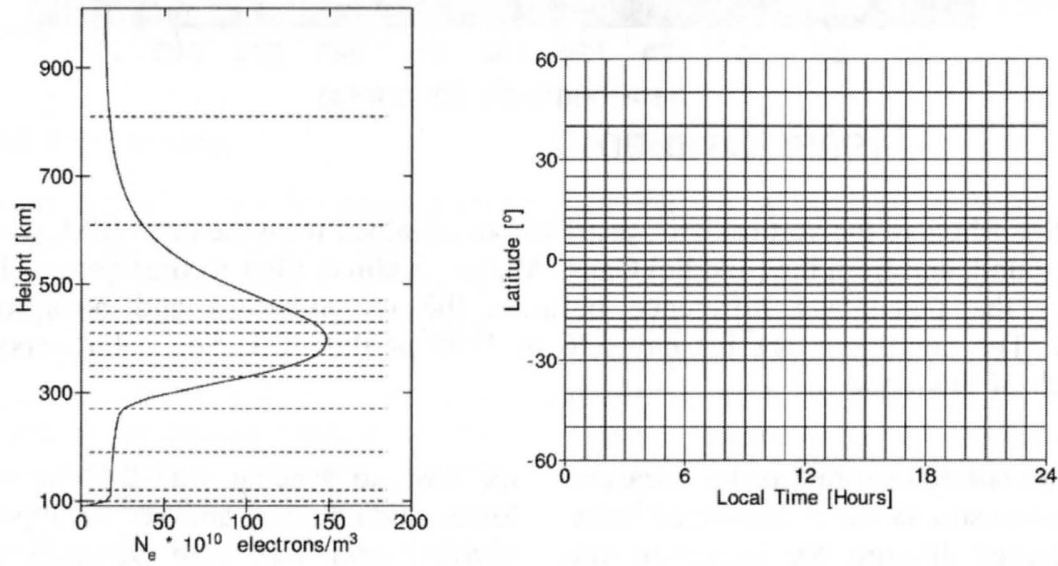

Figure 8. The nonuniform spatial grid used in the Gauss-Markov Kalman filter. 

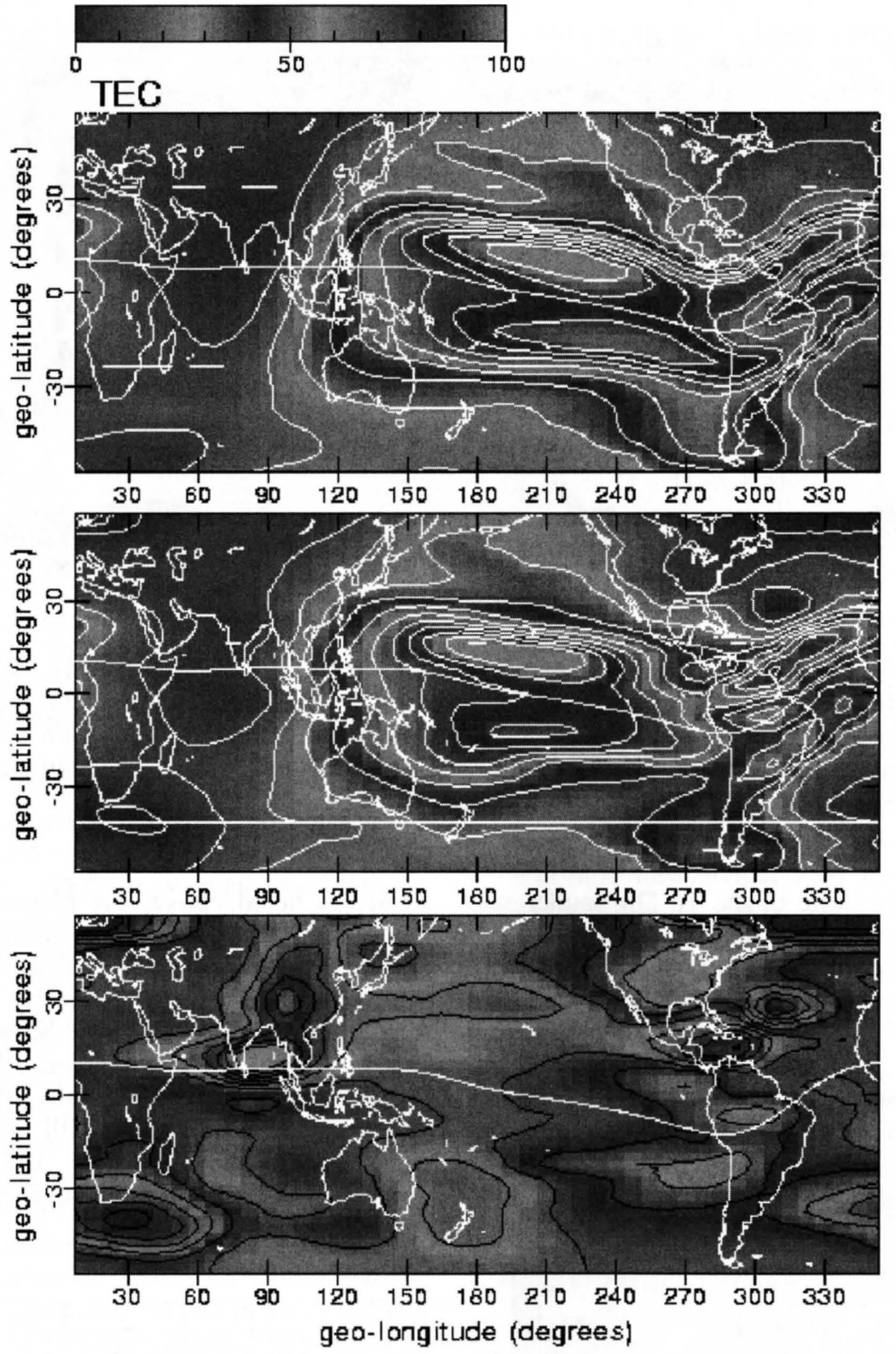

1998/336 0:00

Figure 9. Snapshots of the vertical TEC distributions obtained from the (top) IFM, which is used as the background, and from the (middle) Gauss-Markov Kalman filter reconstruction. The bottom panel shows the percentage difference between the reconstructed and background TEC distributions. The conditions are for day 336 in 1998 at 0:00 UT. See color version of this figure at back of this issue.

filter then provides the corrections due to the measurements. For the $\mathrm{N}_{\mathrm{e}}$ reconstructions, a Sun-fixed reference frame was adopted (Figure 8). However, this reference frame was adaptive and nonuniform. The longitude spacing was fixed at a 1-hour interval, but the latitude spacing was $2.5^{\circ}$ at low latitudes, $5^{\circ}$ at lower midlatitudes, and $10^{\circ}$ at upper midlatitudes. The vertical grid was also nonuniform, with a $20-\mathrm{km}$ resolution in the vicinity of $h_{m} F_{2}$. The vertical grid was adaptive in that as the Earth rotated, the vertical 
grid above a given location moved up and down, tracking the movement of $h_{m} F_{2}$.

[31] Figure 9 shows a snapshot of the Gauss-Markov Kalman filter reconstruction of the global TEC distribution from $60^{\circ} \mathrm{S}$ to $60^{\circ} \mathrm{N}$ at 0:00 UT on day 336 in 1998 . The measurements from the 2 DMSP satellites, 42 ground-based receivers, and 1 Digisonde (Wallops Island) were continuously assimilated in the filter over the 3-day period at a 15-min interval starting at 0:00 UT on 2 December 1998. The vertical TEC was then calculated by integrating through the reconstructed three-dimensional $\mathrm{N}_{\mathrm{e}}$ distribution from 90 to $3000 \mathrm{~km}$ (upper boundary). The top panel in Figure 9 shows the background (IFM run with no data assimilation) and the bottom panel shows the difference between the TEC distributions (reconstructed minus background). The difference is shown as a percentage, with green no change, red enhancements, and blue depletions. Clearly, the assimilation of measurements via a Kalman filter leads to a significantly different TEC distribution, and these results are currently being validated.

\section{Physics-Based Kalman Filter Reconstruction}

[32] Our physics-based ionosphere-plasmasphere model is now being used in the Kalman filter, but in the reconstruction that is shown in a companion paper [Scherliess et al., 2004] only synthetic data were used. The example was for a localized region in South America and the geophysical conditions were for December solstice, $F_{10.7}=150$, and quiet geomagnetic activity $\left(K_{p}=2\right)$. Two types of synthetic data were used, including three GPS ground receivers and one Digisonde at the magnetic equator. The synthetic data were assimilated at a 15-min interval. For this example, all three levels of GAIM were run simultaneously for a 1-day period. Further details concerning this example are given in the companion paper by Scherliess et al. [2004].

\section{Summary and Discussion}

[33] GAIM uses a time-dependent physics-based model of the global ionosphere-plasmasphere system and a Kalman filter as a basis for assimilating a diverse set of real-time (or near real-time) measurements. When completed, GAIM will provide specifications and forecasts on a spatial grid that can be global, regional, or local. This model is still under development, and in this paper we presented an overview of our basic approach and we described the current status of the model.

[34] Because GAIM will be an extensive model with many different algorithms, the model is being constructed in a step-by-step procedure. In the initial development phase, the physics-based ionosphere-plasmasphere model was not used in the Kalman filter. Instead, an ionospheric model was used to provide a background ionosphere and a relatively simple GaussMarkov process was used with the Kalman filter to describe the change in electron density, $\Delta \mathrm{Ne}$, due to the measurements. Our first study with this simplified GAIM model involved synthetic data (with noise) and this study verified that our Kalman filter algorithms were working properly [Schunk et al., 2003]. Next, the synthetic data were replaced with real data, but the Gauss-Markov process was still used. This work was described in section 3 of this paper. The third step of our model construction involved the use of a physics-based ionosphere-plasmasphere model in the Kalman filter, but synthetic data were used. This effort was briefly described in section 4 of this paper and is described in more detail in a companion paper by Scherliess et al. [2004]. Currently, we are in the process of using real measurements in the physics-based Kalman filter model, and this work will be presented in the near future.

[35] Data assimilation models like GAIM are under development for the ionosphere-thermosphere area and it is clear that they will have a major impact on the field during the coming decade. With a physics-based Kalman filter model of the ionosphere assimilating millions of measurements per day, global ionospheric reconstructions will be available on a continuous basis day after day throughout the year. With these reconstructions, the operational community should be able to produce reliable products for a range of applications and the scientific community should be able to resolve a host of long-standing science issues. Consequently, a major advance in ionospheric and thermospheric physics can be anticipated during the coming decade.

[36] Acknowledgments. This research was supported by the DoD MURI program via grant N00014-99-1-0712 to Utah State University.

\section{References}

Anderson, D., A. Anghel, K. Yumoto, M. Ishitsuka, and E. Kudeki (2002), Estimating daytime vertical $\mathrm{E} \times \mathrm{B}$ drift velocities in the equatorial $\mathrm{F}$-region using ground-based magnetometer observations, Geophys. Res. Lett., 29(12), 1596, doi:10.1029/2001GL014562.

Bekerat, H. A., R. W. Schunk, L. Scherliess, and V. Eccles (2001), Determination of ionospheric high-latitude drivers for GAIM using DMSP data, Eos Trans. AGU, 82(47), 958.

Daley, R. (1991), Atmospheric Data Analysis, Cambridge Univ. Press, New York.

Fuller-Rowell, T., C. Minter, and M. Codrescu (2002), Data assimilation for neutral thermospheric species during geo- 
magnetic storms, in 2002 Ionospheric Effects Symposium, pp. 239-247, Off. of Nav. Res., Arlington, Va. (Available as PB2003-104280 from www.ntis.gov)

Hardy, D. A., M. S. Gussenhoven, and. E. Holeman (1985), A statistical model of auroral electron precipitation, J. Geophys. Res., 90, 4229-4248.

Hedin, A. E. (1991), Extension of the MSIS thermospheric model into the middle and lower atmosphere, J. Geophys. Res., 96, 1159-1172.

Hedin, A. E., et al. (1991), Revised global model of thermospheric winds using satellite and ground-based observations, J. Geophys. Res., 96, 7657-7688.

Jee, G., R. W. Schunk, and L. Scherliess (2001), On the determination of the global neutral wind field using TEC observations, Eos. Trans. $A G U, 82(47), 961$.

McCoy, R. P. (2001), Space weather-lessons from the meteorologists, in Space Weather, Geophys. Monogr. Ser., vol. 125, edited by P. Song, H. J. Singer, and G. L. Siscoe, pp. 31-37, AGU, Washington, D.C.

Scherliess, L., and B. G. Fejer (1999), Radar and satellite global equatorial F region vertical drift model, J. Geophys. Res., 104, 6829-6842.

Scherliess, L., R. W. Schunk, J. J. Sojka, and D. C. Thompson (2004), Development of a physics-based reduced state Kalman filter for the ionosphere, Radio Sci., 39, RS1S04, doi:10.1029/2002RS002797, in press.

Schunk, R. W. (2002), An ionosphere-plasmasphere model (IPM), Tech. Rep., Space Environ. Corp., Logan, Utah.
Schunk, R. W., and A. F. Nagy (2000), Ionospheres, Cambridge Univ. Press, New York.

Schunk, R. W., J. J. Sojka, and J. V. Eccles (1997), Expanded capabilities for the ionospheric forecast model, Rep. AFRLVS-HA-TR-98-0001, 1-142.

Schunk, R. W., L. Scherliess, and J. J. Sojka (2003), Recent approaches to modeling ionospheric weather, Adv. Space Res., 31, 819-828.

Titheridge, J. E. (1998), Temperatures in the upper atmosphere and plasmasphere, J. Geophys. Res., 103, 2261-2277.

Weimer, D. R. (1995), Models of the high-latitude electric potential derived with a least error fit of spherical harmonic coefficients, J. Geophys. Res., 100, 19,595-19,608.

D. N. Anderson, M. Codrescu, T. J. Fuller-Rowell, and C. Minter, NOAA/Space Environment Center, Department of Commerce, Boulder, CO 80305, USA.

M. Hairston and R. A. Heelis, W. B. Hanson Center for Space Sciences, University of Texas at Dallas, Richardson, TX 75083, USA.

B. M. Howe, Applied Physics Laboratory, University of Washington, Seattle, WA 98105, USA.

L. Scherliess, R. W. Schunk, J. J. Sojka, and D. C. Thompson, Center for Atmospheric and Space Sciences, Utah State University, Logan, UT 84322, USA. (schunk@cc.usu. edu) 